Alfons Weichenrieder, Eren Gürer

\title{
Inflation, Inflationsmessung und Zentralbankpolitik
}

Inflation ist ein Konstrukt. Sie wird von unterschiedlichen Akteur*innen unterschiedlich wahrgenommen. Zum Teil passiert dies, weil Warenkörbe differieren, zum Teil weil Erwartungen unterschiedlich gebildet werden. Dieser Beitrag diskutiert die Heterogenität der Inflation und ihrer Wahrnehmung und was dies für die Zielgröße der Zentralbankpolitik bedeutet.

\section{Das Inflationsziel der EZB}

Die Europäische Zentralbank (EZB) strebt ein Inflationsziel von nahe, aber unter $2 \%$ an. Dieses Inflationsziel ist nicht in den Europäischen Verträgen verankert. Dort wird in Art. 127 AEUV lediglich das vorrangige EZB-Ziel der Preisstabilität festgeschrieben. Dies bedeutet, dass eine Inflation von $0 \%$ durchaus dem Auftrag der Verträge entspräche. Das Inflationsziel von nahe, aber unter $2 \%$ entspringt der Interpretation des EZB-Rats, wie er in einem Beschluss von 2003 festgehalten wird. Ein ursprünglicher Beschluss des EZB-Rats aus dem Jahr 1998 sprach von einem Inflationsziel von unter $2 \%$.

Auch wenn innerhalb der OECD-Länder ein 2\%-Ziel im Konzert der Zentralbanken nicht ungewöhnlich ist, verbinden sich mit dieser Interpretation des Inflationsziels wirtschaftspolitisch wichtige Implikationen: Während die seit 2014 unter $2 \%$ liegende Inflationsrate nach dem Beschluss von 1998 keine Begründungsbasis für eine ag-

\footnotetext{
(C) Der/die Autor(en) 2020. Open Access: Dieser Artikel wird unter der Creative Commons Namensnennung 4.0 International Lizenz (https:// creativecommons.org/licenses/by/4.0/deed.de) veröffentlicht.
}

Open Access wird durch die ZBW - Leibniz-Informationszentrum Wirtschaft gefördert.

Prof. Dr. Alfons J. Weichenrieder ist Professor für Volkswirtschaftslehre und Finanzwissenschaft an der Goethe-Universität Frankfurt, Gastprofessor an der WU Wien und Forschungsgruppenleiter am Leibniz Institut SAFE.

Eren Gürer, M.Sc., ist wissenschaftlicher Mitarbeiter im Team von Prof. Weichenrieder an der Goethe-Universität Frankfurt. gressive Ausweitung der Zentralbankgeldmenge abgegeben hätte, gibt der Beschluss von 2003 hier mehr Rechtfertigungsspielraum. Dem steht gegenüber, dass an der Nullzinsgrenze, die bereits seit geraumer Zeit erreicht ist, Zweifel existieren, inwieweit die Geldpolitik in der Lage ist, die gesamtwirtschaftliche Nachfrage anzuregen und Preiseffekte zu induzieren.

Begründungen für eine moderate, positive Inflationsrate

Die EZB rechtfertigt das $2 \%$-Ziel mit drei Argumenten:

- Erstens könne eine Zielinflation von nahe $2 \%$ die Gefahr einer Deflation verringern.

- Zweitens sei eine positive durchschnittliche Inflationsrate hilfreich, um zu vermeiden, dass in einem heterogenen Euroraum einige Länder und Regionen eine negative Inflation erreichen.

- Drittens könne die Messung der Inflation mit kleinen, aber systematischen Messfehlern einhergehen. Falls dem so ist, könnte eine gemessene Inflation von null schon eine Deflation bedeuten.

Jenseits dieser offiziellen, auch auf der EZB-Homepage aufgeführten Gründe für moderate Inflation, werden in der Literatur zahlreiche zusätzliche Argumente diskutiert (Diercks, 2019). Unter Ökonominnen scheinen insbesondere zwei Argumente starkes Gewicht zu besitzen:

- Spätestens seit Tobin (1972) lautet ein weitverbreitetes und geteiltes Argument, dass die Effizienz von Arbeitsmärkten von einer positiven Inflation profitiert, weil Arbeitnehmer*innen eine hohe Aversion gegenüber Nominallohnsenkungen besitzen. Trifft eine solche Nominallohnrigidität zu, bedarf es einer Inflation, um in bestimmten Branchen oder Teilen der Wirtschaft, die von einer negativen Produktivitätsentwicklung betroffen sind, eine Reallohnminderung und damit den Erhalt von Arbeitsplätzen sowie einen organischen Wirtschaftswandel zu erreichen.

- Ein weiteres, breit vertretenes Argument für moderate Inflation ist, dass Zinsen nahe der Nullzinsgrenze die Fähigkeit von Notenbanken stark einschränken, durch weitere Zinssenkungen zu einer Stimulierung der Ökonomie beizutragen. Eine solche Situation ist 
Abbildung 1

Konsumentenpreisindex (HVPI) und BIP-Deflator

Jährliche Änderung in \%
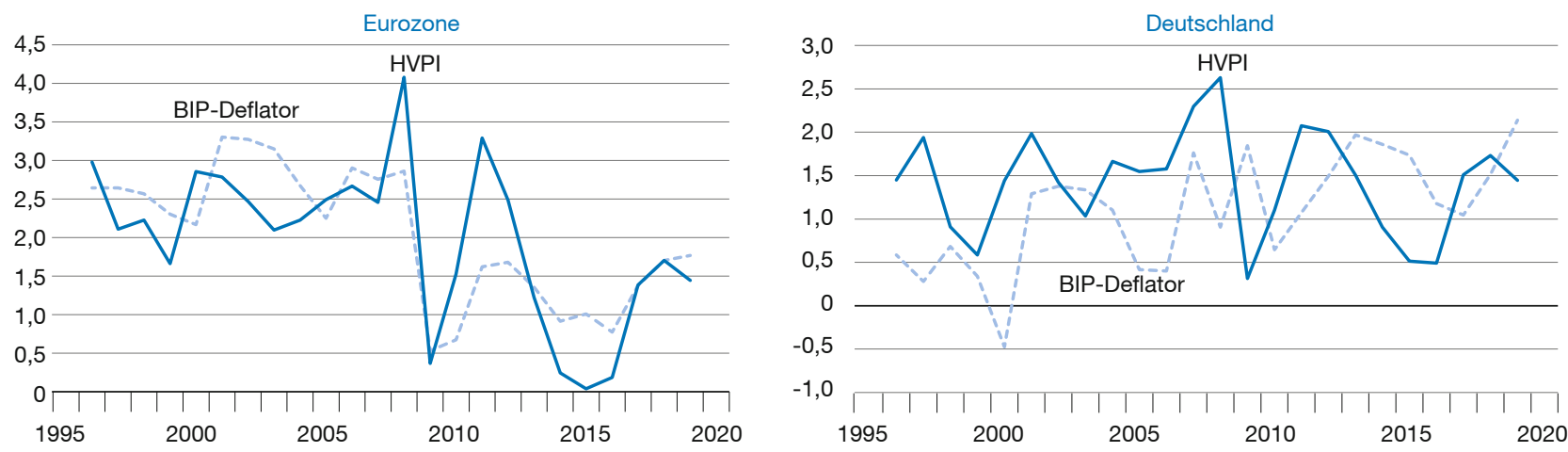

Quelle: Weltbank, eigene Berechnungen.

in der Eurozone seit 2014 eingetreten. Zwar wurden verstärkt Anstrengungen unternommen, den Nominalzins doch unter den Nullzins zu drücken. Jedoch sind weitere Zinssenkungen schwierig, weil den Markteilnehmer*innen die Flucht in Bargeld möglich ist, was einem Zins von null verspricht. Zumindest ist dies der Fall, wenn man von Bargeldhaltungskosten, die den effektiven Nominalzins verringern können, abstrahiert. Ein positives Inflationsziel kann vor diesem Hintergrund hilfreich sein, die Gefahr einer Handlungsunfähigkeit der Zentralbank durch eine bindende Zinsuntergrenze zu vermeiden.

\section{BIP-Deflator oder Konsumentenpreisindex?}

So wenig der Vertrag über die Arbeitsweise der EU (AEUV) explizit definiert, welche Inflationsrate noch als preisstabil gelten darf, genauso wenig gibt er vor, welche Definition der Preissteigerungsrate Verwendung finden soll.

Eine Möglichkeit wäre es, den Deflator der inländischen Produktion (BIP) zu verwenden. Dafür spricht, dass es wichtiger ist, eine maßvolle Inflation bei der heimischen Produktion zuzulassen als beim Konsum, um die Probleme inflexibler Löhne auf dem Arbeitsmarkt zu mildern. Ist der nominale Lohn nach unten inflexibel, so kann aus der Sicht der Unternehmen ein negativer Produktivitätsschock besser verarbeitet werden, wenn die Preise der eigenen Produkte im Zeitablauf steigen. Gewinnorientierte Unternehmen setzen den Nominallohn bei Beschäftigungsentscheidungen nämlich nicht in Relation zum allgemeinen Konsumentenpreisniveau, sondern zum Preisniveau ihres eigenen Produktionsoutputs.

Die Europäische Zentralbank verwendet für ihre Politikentscheidungen den harmonisierten Konsumentenpreis-

1 Aber auch hier kommt dem BIP-Deflator möglicherweise eine hilfreiche Indikatorrolle zu, weil es für die konjunkturelle Entwicklung wichtig ist, ob die Käufe inländischer Güter aufgeschoben werden oder „nur" die Käufe von Importen.

index (HVPI). Dafür werden die Kostenänderungen zum Erwerb von typischen nationalen Konsumbündeln auf das Niveau der Eurozone aggregiert. Für die Orientierung am Konsumentenpreisindex sprechen auf den ersten Blick bestimmte Aspekte der Deflationsgefahr. Ein Problem der Deflation könnte sein, dass Konsument*innen in Erwartung weiter sinkender Preise Konsumgüterkäufe aufschieben und dadurch ein Abschwung selbstverstärkende Tendenzen erhielte. Diese Gefahr wird eher gebannt, wenn es in Normalzeiten einen stetigen Anstieg des Konsumentenpreisniveaus gibt. ${ }^{1}$

Eine Deflation birgt auch Gefahren in Kombination mit nominal rigiden Löhnen. In diesem Fall führen sinkende Erzeugerpreise zu einer indirekten Reallohnerhöhung. Ein laufender Anstieg des BIP-Deflators könnte deshalb auch im Hinblick auf Deflationsgefahr eine geeignetere Richtschnur sein als der laufende Anstieg des Konsumentenpreisindex.

Darüber hinaus beinhaltet der BIP-Deflator auch Preissteigerungen bei Baudienstleistungen und kann daher prinzipiell auch Kostenerhöhungen beim selbstgenutzten Wohnungseigentum widerspiegeln, sofern diese nicht nur auf höhere Grundstückspreise zurückzuführen sind. Auch in einer Ökonomie wie der deutschen, die tendenziell auf die Produktion von Investitionsgütern spezialisiert ist, kann der alleinige Fokus auf den Konsumentenpreisindex in die Irre führen. 


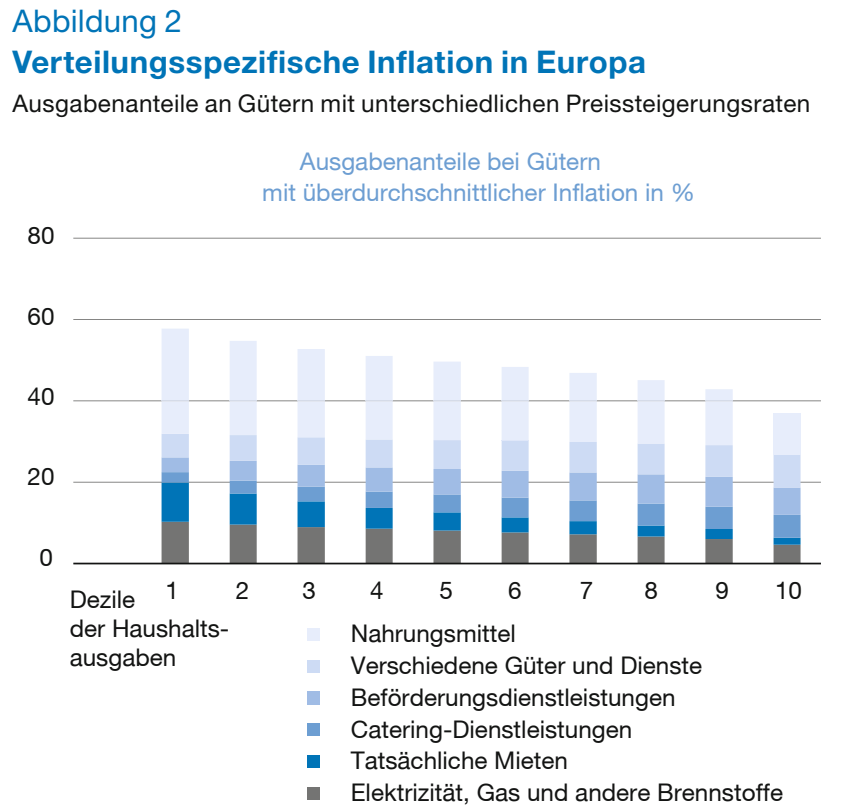

Ausgabenanteile bei Gütern mit unterdurchschnittlicher Inflation in \%

30

20

10

0

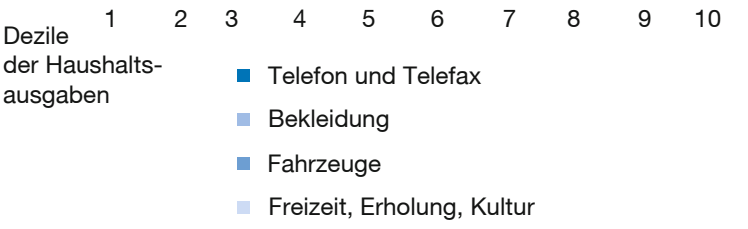

Anmerkung: Abbildung basiert auf den ungewichteten Durchschnitten aus 25 EU-Ländern im Zeitraum 2001 bis 2015.

Quelle: Gürer und Weichenrieder (2020).

Langfristig gleichen sich die Unterschiede zwischen dem Konsumentenpreisindex und dem BIP-Deflator immer wieder aus. Zeitlich befristet indes existieren deutliche Differenzen. Abbildung 1 zeigt beispielhaft die Unterschiede für den Euroraum und Deutschland. So lag in den sogenannten Nullerjahren, in denen Deutschland eine gewisse Lohnzurückhaltung und zunehmende Wettbewerbsfähigkeit an den Tag legte, der BIP-Deflator meist unter dem Konsumentenpreisindex. Für den Euroraum ergibt sich nach der Finanzkrise eine weniger schwankende Inflationsrate, wenn man auf den BIP-Deflator sieht. Insbesondere im Jahr 2015 lag eine so deutliche Differenz zwischen der niedrigen Inflation der Konsumentenpreise und der höheren Steigerungsrate des BIP-Deflators, dass einige Ökonom*innen die EZB kritisierten, diese würde eine Deflation bekämpfen, die bei einem Blick auf den BIP-Deflator gar nicht existiere (Alcidi, Busse und Gros, 2016). Auch in der ursprünglichen Arbeit zur Taylor-Regel der Zentralbankpolitik wird interessanterweise vom BIPDeflator ausgegangen, nicht vom Konsumentenpreisindex (Taylor, 1993).

\section{Die Heterogenität der \\ Konsumentenpreissteigerungen}

Die Preisentwicklung eines Konsumentenkorbs hängt von dessen Zusammensetzung ab, und ärmere Haushalte haben andere Konsumgewohnheiten als reichere Haushalte. Gerade in Entwicklungsländern gibt es zahl- reiche Forschungsarbeiten, die zeigen, dass die Inflation für unterschiedliche Einkommensgruppen signifikant differieren kann (Arndt, Jones und Salvucci, 2015; Beck, 2015). Aber auch für viele EU-Länder zeigen die Daten der Jahre 2001 bis 2015, dass sich die haushaltsspezifischen Inflationsraten signifikant unterscheiden. Haushalte mit geringen Gesamtausgaben hatten deutlich höhere Inflationsraten als einkommensstärkere Haushalte. Im Durchschnitt über 25 EU-Länder ergibt sich, dass die jährliche Inflationsrate für das unterste Dezil des jeweiligen Landes ca. 0,8 Prozentpunkte höher war als für das oberste Dezil (Gürer und Weichenrieder, 2020). Dies ist in den einzelnen Ländern meist über die höheren Preissteigerungsraten von Mieten, Energie und Nahrungsmitteln begründet, die bei ärmeren Haushalten einen höheren Anteil an den Haushaltsausgaben haben als bei reicheren. Die Abbildung 2 illustriert die verschiedenen Ausgabenanteile, getrennt nach den Dezilen der Gesamtausgaben. Dezile mit höheren Nummern tätigen höhere absolute Konsumausgaben. Die linke Abbildung stellt Käufe dar, bei denen im Durchschnitt höhere Preissteigerungsraten vorlagen, die rechte Abbildung mit unterdurchschnittlichen Preissteigerungen. Reichere Dezile von Haushalten konsumieren eher die Güter und Dienstleistungen mit niedrigeren Preissteigerungen, ärmere eher solche mit überdurchschnittlichen Preissteigerungen. Die Inflation begünstigte für den betrachteten Zeitraum daher reichere Haushalte. Die Erfahrungen der vergangenen Jahre müssen sich nicht unbedingt fortsetzen. Aber sie 
verdeutlichen, dass es die eine Preissteigerungsrate der Konsumausgaben nicht gibt.

Bis zu einem gewissen Grade wird der Diversität der Inflationsraten auch durch die EZB Rechnung getragen. In zahlreichen Kommunikationen wird neben dem HVPI auch auf die Kerninflation abgestellt, in die die meist stärkeren Schwankungen bei Nahrungsmittel- und Energiepreisen nicht eingehen. Gerade die Entwicklung der Nahrungsmittelpreise kann jedoch unter Umständen in besonders starkem Maße die gefühlte Inflation der Verbraucher ${ }^{\star} i n n e n$ beeinflussen. Güter, die sehr oft gekauft werden, dürften einen größeren Einfluss auf die gefühlte Inflation besitzen (Hintze, 2005). Vor diesem Hintergrund gibt es daher auch das Konzept einer Inflationsrate, die nur Güter beinhaltet, welche häufig gekauft und nicht über Daueraufträge bezahlt werden - Frequent out of Pocket Purchases (FROOP), deren Preise die FROOP-Inflation bestimmen. In der Tat ist die FROOPInflation im Euroraum konsistent höher als die offizielle Inflationsrate (Arioli et al., 2017).

Umfrageergebnisse zeigen darüber hinaus, dass auch die gefühlte Inflation im Euroraum deutlich und konsistent über der offiziellen HVPI-Steigerungsrate liegen. Für Frauen, die immer noch häufiger Dinge des täglichen Konsums kaufen dürften, liegt die gefühlte Inflation noch etwas weiter jenseits der offiziellen Preissteigerungsrate. Allerdings kann die FROOP-Inflation nur einen kleinen Teil der zu hoch wahrgenommenen Inflation erklären (Arioli et al., 2017). Schlägt sich die individuell gefühlte Inflation in Inflationserwartungen nieder, so sind auch reale Effekte möglich. So scheinen sich höhere Inflationserwartungen durchaus in geringerer Ersparnis niederzuschlagen. ${ }^{2}$

\section{Vermögensgüterpreise und selbstgenutztes Wohneigentum}

Die Frage, inwieweit unterschiedliche Inflationsraten für unterschiedliche Einkommensgruppen berücksichtigt werden sollten, wird für die Geldpolitik kaum erörtert. Dies ist insofern auch verständlich, als die EZB keine adäquaten Instrumente hat, um die sektorale Struktur der Inflation zu beeinflussen, und auch kein spezielles Mandat. In der Debatte um das richtige Inflationsmaß wird eher diskutiert, inwiefern Vermögensgüterpreise in die Inflationsmessung miteinbezogen werden sollten. Weil der HVPI die Kosten von Konsumgütern abdeckt, ist die Vernachlässigung von Vermögensgegenständen folgerichtig. Davon unbenommen würde eine Einbeziehung der Preissteigerungen von Vermögensgegenständen Ausweitungen der Geldmenge, die ohne reale Effekte verpuffen, besser indizieren. Zudem ist kontrovers, ob die Preise selbstgenutzten Wohneigentums nicht doch in den Konsumentenpreis eingehen sollten. Derzeit ist dies bei der Berechnung des HVPI im Euroraum nicht der Fall.

In Ländern, die das selbstgenutzte Wohneigentum berücksichtigen, kommen drei verschiedene Konzepte zur Anwendung (Eurostat, 2017, Abschnitt 1.3). Beim Anschaffungskostenkonzept wird Wohneigentum in der Berechnung des VPI ähnlich berücksichtigt wie andere langlebige Konsumgüter (Autos, Waschmaschinen): Die Ausgaben für Neubauten bzw. Wohnungen, die nicht nur zwischen Konsument*innen verkauft werden, gehen zum Zeitpunkt des Kaufs ein, eine Periodisierung der Ausgaben unterbleibt. Australien, Neuseeland und Finnland nutzen dieses Konzept für die Messung ihrer nationalen Inflationsrate. Die USA, Japan, Dänemark, Norwegen, die Schweiz, die Tschechische Republik, Mexiko und Südafrika unterstellen bei selbstgenutztem Wohneigentum die Miete eines gleichwertigen Objekts (Mietäquivalenzansatz). ${ }^{3}$ Beim Nutzungskostenkonzept, das in Kanada, Island und Schweden angewandt wird, werden nur die Wohnnebenkosten (Instandhaltungskosten, Versicherung etc.) in die Preissteigerungsrate miteinbezogen.

Weil selbstgenutztes Wohneigentum ökonomisch als Versicherungsinstrument gegen Preissteigerungen im Mietmarkt verstanden werden kann, lässt sich einwenden, dass der Mietäquivalenzansatz über das Ziel hinausschießt. Dies spricht eher für das Anschaffungskostenkonzept oder aber eine Ausrichtung auf den BIP-Deflator.

\section{Heterogene Inflation und ihre Politikimplikation}

Befürwortet man im Hinblick auf den Strukturwandel bei nominal rigiden Löhnen eine moderate Inflation, etwa von $2 \%$, so erscheint vor dem Hintergrund der obigen Diskussion eine verstärkte Betrachtung des BIP-Deflators als Hintergrund geldpolitischer Entscheidungen sinnvoll. Im Hinblick auf die Beschreibung der Wohlfahrtsperspektive ist dem Konsumentenpreisindex der Vorzug zu geben.

Die Inflation hat temporär für verschiedene Einkommensgruppen eine unterschiedliche Höhe. So sehr es adäquat erscheint, diese Unterschiede ehrlich zu kommunizieren, so wenig sinnvoll ist es, die Betrachtung alternativer Inflationsmaße mit einer Ausweitung des politischen Mandats der Notenbank zu verbinden. Ähnlich wie in der Um- 
weltpolitik haben nationale Regierungen und die Legislative die besseren Instrumente und die bessere politische Legitimation, um begleitende Umverteilungsmaßnahmen durchzuführen.

\section{Literatur}

Alcidi, C., M. Busse und D. Gros (2016), Is there a need for additional monetary stimulus? Insights from the original Taylor Rule, CEPS Policy Brief, Nr. 342, April.

Arioli, R., C. Bates, H. Dieden, I. Duca, R. Friz, C. Gayer, G. Kenny, A. Meyler und I. Pavlova (2017), EU consumers' quantitative inflation perceptions and expectations: An evaluation, ECB Occasional Paper, Nr. 186.

Arndt, C., S. Jones und V. Salvucci (2015), When do relative prices matter for measuring income inequality? The case of food prices in Mozambique, Journal of Economic Inequality, 13(3), 449-464.
Beck, U. (2015), Keep it real: Measuring real inequality using survey data from developing countries, WIDER Working Paper, Nr. 2015/133.

Diercks, A. M. (2019), The reader's guide to optimal monetary policy, Federal Reserve Board, Working Paper.

Eurostat (2017), Technical manual on owner-occupied housing and house price indices, https://ec.europa.eu/eurostat/documents/7590317/0/ Technical-Manual-OOH-HPI-2017/ (23. Oktober 2020).

Gürer, E. und A. J. Weichenrieder (2020), Pro-rich inflation in Europe: Implications for the measurement of inequality, German Economic Review, 21, 107-138.

Hill, R. J., M. Steurer und S. R. Waltl (2019), Owner-occupied housing, inflation, and monetary policy, Graz Economics Papers, 2019-05.

Hinze, J. (2005), Gefühlte Inflation, Wirtschaftsdienst, 85(12), 800-801.

Taylor, J. (1993), Discretion versus policy rules in practice, Carnegie-Rochester Conference Series on Public Policy, 39, 195-214.

Tobin J. (1972), Inflation and unemployment, American Economic Review, $62,1-18$.

Vellekoop, N. und M. Wiederholt (2019), Inflation expectations and choices of households, SAFE Working Paper, Nr. 250. 